\title{
Dual barrier-projection and barrier-Newton methods in linear programming
}

\author{
Vitali G. Zhadan \\ Computing Center of the Russian Academy of Sciences \\ 40 Vavilov Str., 117967 Moscow GSP-1, Russia. Tel: (095)-135-25-39. \\ Fax: (095)-135-61-59. e-mail: zhadan@ccas.rus
}

\begin{abstract}
In this paper the stable version of dual barrier-projection method and the dual barrierNewton method for solving linear programming problems are proposed. These methods are obtained by using space transformation techniques which are applied for releasing from nonnegativity of dual slack variables. The continuous and discrete versions of both methods are considered and their local convergence is investigated. A modification of the dual barrier-projection method is proposed in which a steepest descent is utilized.
\end{abstract}

\section{Keywords}

Linear programming, dual problem, space transformation, gradient projection method, Newton's method, steepest descent

\section{INTRODUCTION}

The paper deals with dual algorithms for solving linear programming problems. These algorithms are similar to primal ones which were proposed by Evtusenko and Zhadan $(1978,1994)$ and which are based on space transformation techniques. Using a surjective transformation we convert the original linear programming problem with nonnegative variables to a new problem where variables belong to the whole space. We can apply various numerical methods for solving this new problem, in particular, the stable version of the gradient projection method (Tanabe, 1980) and the Newton method. After an inverse transformation to the original space a family of barrier-projection and barrierNewton methods are obtained. As a result of a space transformation additional diagonal matrices appear in the right-hand sides of formulas describing methods. These matrices prevent trajectories from leaving the positive orthant of the space. Different numerical methods are obtained by different choices of the space transformation.

This paper is devoted to dual barrier-projection and barrier-Newton methods. The space transformation technique is applied there for dual slack variables. In section 2 we outline the basic approach and propose a family of dual barrier-projection methods. These 
methods are described by systems of ordinary differential equations. Numerical algorithms are obtained as discretization of dynamical systems. In the case of exponential space transformation these methods are similar to the dual affine scaling algorithm proposed by Adler, Karmarkar, Resende and Veiga (1989). We show that if the quadratic space transformation is used then we obtain an exponential rate of convergence for continuous methods and a linear rate of convergence for discrete versions.

In section 3 we consider a variant of the dual barrier-projection method with a steepest descent which converges to the solution of the dual problem in a finite number of iterations. In section 4 we propose the barrier-Newton method.

\section{DUAL BARRIER-PROJECTION METHOD}

We consider a linear programming problem

$$
\begin{aligned}
\operatorname{minimize} & c^{T} x \\
\text { subject to } & A x=b \\
x & \geq 0_{n},
\end{aligned}
$$

and its dual

$$
\text { maximize } b^{T} u
$$

subject to $A^{T} u \leq c$,

where $A$ is a full rank $m \times n$ matrix where $m<n, x$ and $c$ are $n$-dimensional vectors, and $b$ and $u$ are $m$-dimensional vectors.

We define the set of feasible solutions of dual problem (2) and its interior

$U=\left\{u \in R^{m}: v=c-A^{T} u \geq 0_{n}\right\}, \quad U_{0}=\left\{u \in R^{m}: v=c-A^{T} u>0_{n}\right\}$.

Throughout the paper we assume that $U_{0}$ is nonempty and that the primal and dual nondegeneracy holds.

Let $v=\varphi(w)$ be a componentwise space transformation from $R^{n}$ to the nonnegative orthant $R_{+}^{n}$, i.e. $v^{i}=\varphi^{i}\left(w^{i}\right), 1 \leq i \leq n$. Suppose also that this transformation is differentiable and is such that the closure of $\varphi\left(R^{n}\right)$ coincides with $R_{+}^{n}$. In this case every vector in $R_{+}^{n}$ is the image of at least one vector in $R^{n}$ or it is the image of a limit point of a sequence in $R^{n}$.

Let $w^{i}=\psi^{i}\left(v^{i}\right)$ denote the inverse transformation of $\varphi^{i}\left(w^{i}\right)$. This transformation exists at least in a neighborhood of a point $v_{0}^{i}=\varphi^{i}\left(w_{0}^{i}\right)$, as long as $\dot{\varphi}^{i}\left(w_{0}^{i}\right) \neq 0$. If $\tilde{J}(w)$ is the Jacobian matrix of the transformation $\varphi(w)$, then the Gram matrix $G(v)=\tilde{J}(\psi(v)) \tilde{J}^{\top}(\psi(v))$ is diagonal, i.e.

$G(v)=D(\theta(v)), \quad \theta(v)=\left[\theta^{1}\left(v^{1}\right), \theta^{2}\left(v^{2}\right), \ldots, \theta^{n}\left(v^{n}\right)\right]$,

where $\theta^{i}\left(v^{i}\right)=\left(\gamma^{i}\left(v^{i}\right)\right)^{2}, \gamma^{i}\left(v^{i}\right)=\dot{\varphi}^{i}\left(\psi^{i}\left(v^{i}\right)\right), 1 \leq i \leq n$.

We impose the following conditions on the space transformation $\varphi(w)$ :

$C_{1}$. The functions $\theta^{i}\left(v^{i}\right)$ are defined and continuous in some neighborhood of $R_{+}^{1}$ and $\theta^{i}\left(v^{i}\right)=0$ if and only if $v^{i}=0$, where $1 \leq i \leq n$. 
$C_{2}$. The functions $\theta^{i}\left(v^{i}\right)$ are continuously differentiable in some neighborhood of $R_{+}^{1}$ and $\dot{\theta}^{i}(0)>0,1 \leq i \leq n$.

Here we consider only two simple surjective transformations:

$v=\frac{1}{4} D(w)(w), \quad v=e^{-w}$,

where the $i$-th component of the $n$-vector $e^{-w}$ is $e^{-w^{i}}$. For these transformations we obtain, respectively

$\theta(v)=v, \quad G(v)=D(v) ; \quad \theta(v)=D(v) v, \quad G(v)=D^{2}(v)$.

In both cases the Gram matrix is singular on the boundary of $R_{+}^{n}$. These transformations satisfy $C_{1}$. Condition $C_{2}$ holds only for the first quadratic transformation (3).

By extension of the space and by converting the inequality constraints to equalities, we transform the original dual problem (2) into the following equivalent problem in the extended space

$$
\begin{array}{cc}
\text { maximize } & b^{T} u \\
\text { subject to } & \varphi(w)+A^{T} u-c=0_{n} .
\end{array}
$$

The Lagrangian associated with this problem is defined by

$\tilde{L}(u, w, x)=b^{T} u+x^{T}\left[\varphi(w)+A^{T} u-c\right]$.

For solving problem (4) we use the stable version of the gradient projection method which is proposed by Tanabe (1980). The method is stated as an initial-value problem involving the following system of ordinary differential equations

$\frac{d u}{d t}=\tilde{L}_{u}(u, w, x(u, w)), \quad \frac{d w}{d t}=\tilde{L}_{w}(u, w, x(u, w))$.

The function $x(u, w)$ is chosen to satisfy the following condition

$\tilde{L}_{x u}(u, w, x) \dot{u}+\tilde{L}_{x w}(u, w, x) \dot{w}=-\tau \tilde{L}_{x}(u, w, x)$.

Since $\dot{v}=\varphi_{w} \dot{w},(5)$ can be rewritten in terms of $u$ and $v$ as follows

$\frac{d u}{d t}=b-A x(u, v), \quad \frac{d v}{d t}=-G(v) x(u, v)$,

where $\Phi(v) x(u, v)=A^{T} b+\tau\left(v+A^{T} u-c\right)$ and $\Phi(v)=G(v)+A^{T} A$.

Denote $v(u)=c-A^{T} u$.

Proposition 1 Let the space transformation $\varphi(w)$ satisfy $C_{1}$. Then the matrix $\Phi(v(u))$ is positive definite for all $u \in U_{0}$.

Proposition 2 If all extreme points of the bounded set $U$ are nondegenerate, then $\Phi(v(u))$ is positive definite for any $u \in U$. 
Let $\left[u\left(t, z_{0}\right), v\left(t, z_{0}\right)\right]$ denote the solution of the Cauchy problem (7) with initial condition $u\left(0, z_{0}\right)=u_{0}, v\left(0, z_{0}\right)=v_{0}, z_{0}^{T}=\left[u_{0}^{T}, v_{0}^{T}\right]$. This system of differential equations has the first integral

$v\left(\dot{t}, z_{0}\right)+A^{T} u\left(t, z_{0}\right)-c=\left(v_{0}+A^{T} u_{0}-c\right) e^{-\tau t}$.

Thus, if the initial point $z_{0}$ is such that $v_{0}=v\left(u_{0}\right)$, then $v\left(t, z_{0}\right) \equiv c-A^{T} u\left(t, u_{0}\right)$ for all $t \geq 0$. In this case the system ( 7 ) can be simplified

$\frac{d u}{d t}=b-A x(u), \quad\left(G(v)+A^{T} A\right) x(u)=A^{T} b$,

where $u\left(0, u_{0}\right)=u_{0} \in U$. For this system we obtain the following inequality

$b^{T} \frac{d u}{d t}=\|b-A x(u)\|^{2}+x^{T}(u) G(v(u)) x(u) \geq 0$.

Hence the objective function of the dual problem monotonically increases on a feasible set.

Numerical algorithms are obtained as discretization of dynamical systiems (7), (8). By applying the Euler numerical integration method we obtain the following iterative algorithm

$u_{k+1}=u_{k}+\alpha_{k}\left(b-A x_{k}\right), \quad v_{k+1}=v_{k}-\alpha_{k} G\left(v_{k}\right) x_{k}$,
$\left(G\left(v_{k}\right)+A^{T} A\right) x_{k}=A^{T} b+\tau\left(v_{k}+A^{T} u_{k}-c\right)$.

Similarly for the system (8) we have

$u_{k+1}=u_{k}+\alpha_{k}\left(b-A x_{k}\right), \quad\left(G\left(v_{k}\right)+A^{T} A\right) x_{k}=A^{T} b$,

where $v_{k}=v\left(u_{k}\right)$. Both variants solve the primal and dual problems simultaneously.

Theorem 1 Let $x_{*}$ and $u_{*}$ be unique nondegenerate solutions of Problems (1) and (2), respectively, and let $v_{*}=c-A^{T} u_{*}$. Assume that the space transformation $\varphi(w)$ satisfies conditions $C_{1}, C_{2}$ and $\tau>0$. Then the following statements are true:

1. The pair $\left[u_{*}, v_{*}\right]$ is an asymptotically stable equilibrium state of system (7).

2. The solutions $u\left(t, z_{0}\right), v\left(t, z_{0}\right)$ of system $(\gamma)$ converge locally to the pair $\left[u_{*}, v_{*}\right]$. The corresponding function $x\left(u\left(t, z_{0}\right), v\left(t, z_{0}\right)\right)$ converges to the optimal solution $x_{*}$ of the primal problem (1).

3. The point $u_{*}$ is an asymptotically stable equilibrium state of system (8).

4. The solutions $u\left(t, u_{0}\right)$ of system (8) converge locally to the optimal solution $u_{*}$ of the dual problem (2). The corresponding function $x\left(u\left(t, u_{0}\right)\right)$ converges to the optimal solution $x_{*}$ of the primal problem (1).

5. There exists an $\alpha_{*}>0$ such that for any fixed $0<\alpha_{k}<\alpha_{*}$ the sequence $\left\{u_{k}, v_{k}\right\}$ generated by (10) converges locally with a linear rate to $\left[u_{*}, v_{*}\right]$ while the corresponding sequence $\left\{x_{k}\right\}$ converges to $x_{*}$. 
6. There exists an $\alpha_{*}>0$ such that for any fixed $0<\alpha_{k}<\alpha_{*}$ the sequence $\left\{u_{k}\right\}$ generated by (11) converges locally with a linear rate to $u_{*}$ while the corresponding sequence $\left\{x_{k}\right\}$ converges to $x_{*}$.

If $u \in U_{0}$, then we can transform (8) to the equivalent system

$\frac{d u}{d t}=\left(I_{m}+A G^{-1}(v) A^{T}\right)^{-1} b$,

where $I_{m}$ is the $m \times m$ identity matrix. In the case where $G(v)=D^{2}(v)$ this method is similar to the dual affine scaling algorithm which was proposed and investigated by Adler, Karmarkar, Resende and Veiga (1989).

\section{STEEPEST DESCENT}

It follows from (9) that in order to maximize the value of the objective function at each iteration in the method (11) we must choose the step length $\alpha_{k}$ as large as possible provided that the point $u_{k+1}$ belongs to the feasible set. This way of stepsize choice leads to a variant of the dual barrier-projection method (11) in which a steepest descent is utilized.

Let $u \in U$ and assume that

$v(u)=\left[\begin{array}{c}v^{B}(u) \\ v^{N}(u)\end{array}\right], \quad v^{B}(u) \in R^{s}, \quad v^{N}(u) \in R^{d}$,

where $v^{B}(u)=0_{s}, v^{N}(u)>0_{d}, d=n-s, s \leq m$. Denote the $m \times s$ matrix determined by the first $s$ columns as $B$ and denote the $m \times d$ matrix determined by the rest columns as $N$. Supposing for simplicity that $G(v)=D(v)$, we can write

$\Phi(v)=\left[\begin{array}{cc}B^{T} B & B^{T} N \\ N^{T} B & D\left(v^{N}\right)+N^{T} N\end{array}\right]$.

If $B$ is a full rank matrix then, using the Frobenious formula, we obtain

$\Phi^{-1}=\left[\begin{array}{cc}Q & -Q B^{T} W N D_{N}^{-1} \\ -D_{N}^{-1} N^{T} W B Q & D_{N}^{-1}-D_{N}^{-1} N^{T}\left[W-W B Q B^{T} W\right] N D_{N}^{-1}\end{array}\right]$,

where $Q=\left(B^{T} W B\right)^{-1}, \quad W=\left(I_{m}+N D_{N}^{-1} N^{T}\right)^{-1}, \quad D_{N}=D\left(v^{N}\right)$

Substituting (13) in (8) yields

$\frac{d u}{d t}=\left[W-W B Q B^{T} W\right] b$.

Moreover, we have

$x(u)=\left[\begin{array}{c}x^{B}(u) \\ x^{N}(u)\end{array}\right], \quad x^{B}(u)=Q B^{T} W b, \quad x^{N}(u)=D_{N}^{-1} N^{T}\left[W-W B Q B^{T} W\right] b$. 
Thus

$a_{i}^{T} \frac{d u}{d t}=0_{s}, \quad i \in J^{B}(u)$

where $a_{i}$ is the $i$-th column of the matrix $A$, and $J^{B}(u)=\left\{1 \leq i \leq n: v^{i}(u)=0\right\}$. We supposed previously that $J^{B}(u)=\{1,2, \ldots, s\}$.

The equality (15) shows that an adhesion property holds and that the trajectory can't leave the boundary of the constraint $a_{i}^{T} u \leq c^{i}$ even if $x^{i}(u)<0$. In order to overcome this drawback we will perturb the righthand side of the method (14). Denote

$J_{-}^{B}(u)=\left\{i \in J^{B}(u): x^{i}(u)<0\right\}, \quad J_{+}^{B}(u)=\left\{i \in J^{B}(u): x^{i}(u) \geq 0\right\}$.

Let $\varepsilon>0$ and let

$y \in S^{B}(u)=\left\{z \in R_{+}^{s}: \sum_{i \in J^{B}(u)} z^{i}=1, z^{i}=0, i \in J_{+}^{B}(u)\right\}$.

We introduce a diagonal matrix $Y$ of order $s$ such that $Y^{2}=D(y)$ and modify the matrix $\Phi(v)$ changing only the left upper submatrix

$\Phi(v)=\left[\begin{array}{cc}B^{T} B+\varepsilon Y^{2} & B^{T} N \\ N^{T} B & D\left(v^{N}\right)+N^{T} N\end{array}\right]$.

Then instead of (14) we obtain

$\frac{d u}{d t}=\left\{W-W B[Q-\varepsilon P] B^{T} W\right\} b$,

where $P=Q Y\left[I_{s}+\varepsilon Y Q Y\right]^{-1} Y Q$. Now equality (15) takes place only for $i \in J^{B}(u)$ such that $y^{i}=0$. Moreover, if $\varepsilon$ is sufficiently small and $y^{i}>0$ for $i \in J_{-}^{B}(u)$, then

$a_{i}^{T} \frac{d u}{d t}>0, \quad i \in J_{-}^{B}(u)$

There are several approaches to choosing the vector $y$ from the set $S^{B}(u)$. The simplest one is the approach where a single index $i_{k} \in J_{-}^{B}\left(u_{k}\right)$ is selected and $y$ is set to be equal to the vector $e_{i_{k}} \in S^{B}\left(u_{k}\right)$ with the components

$e_{i_{k}}^{i}=0, i \neq i_{k}, \quad e_{i_{k}}^{i_{k}}=1$.

In this case the inequality (17) holds for any $\varepsilon>0$.

We denote by $q_{i}$ the $i$-th column of the matrix $Q$ and by $q_{i}^{j}$ the $j$-th element of $q_{i}$. We also introduce a vector

$\Delta=A^{T} W\left[I_{m}-B Q B^{T} W+\left(1+q_{i_{k}}^{i_{k}}\right)^{-1} B q_{i_{k}} q_{i_{k}}^{T} B^{T} W\right] b$

and index sets

$J^{N}(u)=\left\{1 \leq i \leq n: v^{i}(u)>0\right\}, \quad J_{+}^{N}(u)=\left\{i \in J^{N}(u): \Delta^{i}>0\right\}$. 
Let, for simplicity, $\varepsilon=1$. Then there is a variant of the method (11) with a steepest descent which can be written as

$u_{k+1}=u_{k}+\alpha_{k} W\left[I_{m}-B Q B^{T} W+\left(1+q_{i_{k}}^{i_{k}}\right)^{-1} B q_{i_{k}} q_{i_{k}}^{T} B^{T} W\right] b$,

where

$\alpha_{k}=\min _{i \in J_{+}^{N}\left(u_{k}\right)} \frac{v_{k}^{i}}{\Delta_{k}^{i}}$

Theorem 2 Let the set $U$ be bounded and let the function $b^{T} u$ have different values at extreme points of $U$. Then the method (18) solves the problem (2) in a finite number of iterations.

Geometrically, the adding of the matrix $D(y)$ means the shifting of active constraints in dual problem (2). In method (18) we shift only a single active constraint. This simplest variant of the dual method possesses the following property, namely, if the point $u_{k}$ is an extreme point of the set $U$, then the further behavior of the method is the same as a dual simplex method.

\section{DUAL BARRIER-NEWTON METHOD}

The method (11) can be interpreted as a contraction mapping for solving the system of nonlinear equations

$b-A x(u)=0_{m}$,

where the function $x(u)$ is defined from the condition

$\left(D(\theta(v))+A^{T} A\right) x(u)=A^{T} b$.

We can apply the Newton method for this purpose. The continuous version of the Newton method leads to the following system of ordinary differential equations

$\Lambda(u) \frac{d u}{d t}=A x(u)-b, \quad \Lambda(u)=-A \frac{d x}{d u}$.

By differentiating the relation (19) with respect to $u$, we obtain

$-D(\dot{\theta}(v)) D(x) A^{T}+\left(D(\theta(v))+A^{T} A\right) \frac{d x}{d u}=0_{n m}$.

Therefore

$\Lambda(u)=-A\left(D(\theta(v))+A^{T} A\right)^{-1} D(\dot{\theta}(v)) D(x(u)) A^{T}$.

Proposition 3 Let all conditions of Theorem 1 be fulfilled. Then $\Lambda\left(u_{*}\right)$ is a nonsingular matrix. 
Theorem 3 Let all conditions of Theorem 1 hold. Then $u_{*}$ is an asymptotically stable equilibrium point of system (20). If the matrix $\Lambda(u)$ satisfies a Lipschitz condition in a neighborhood of $u_{*}$, then the discrete version

$u_{k+1}=u_{k}+\alpha_{k} \Lambda^{-1}\left(u_{k}\right)\left(A x_{k}-b\right), \quad x_{k}=x\left(u_{k}\right)$,

locally converges with at least linear rate to the point $u_{*}$ if the stepsize $\alpha_{k}$ is fixed and $0<\alpha_{k}<2$. If the matrix $\Lambda(u)$ satisfies a Lipschitz condition in a neighborhood of $u_{*}$ and $\alpha_{k}=1$, then the sequence $\left\{u_{k}\right\}$ converges quadratically to $u_{*}$.

Let us suppose now that we use the quadratic space transformation (3). Then the method (20) can be rewritten as follows

$\frac{d u}{d t}=\left[A\left(D(v(u))+A^{T} A\right)^{-1} D(x(u)) A^{T}\right]^{-1}(b-A x(u))$.

On the set $U_{0}$ the right-hand side of this equation can be simplified

$$
\frac{d u}{d t}=\left[A D(x(u)) D^{-1}(v(u)) A^{T}\right]^{-1} b, \quad x(u)=\left[I_{m}-\left(I_{m}+A D^{-1}(v) A^{T}\right)^{-1}\right] b .
$$

Thus the objective function of the dual problem increases along the Newton direction.

According to Theorem 3 the trajectories of the system (22) converge locally to $u_{*}$. The trajectories of the system (23) converge locally to $u_{*}$ on the set $U_{0}$. The discrete version of the method (22) is similar to (21). It is possible to consider the discrete version of the method (23) using a steepest descent.

\section{ACKNOWLEDGEMENT}

Research supported by the Russian Scientific Fund, grant number 94-01-01379, and partially by the Czech Research Grant Agency, grant number 201/93/0429.

\section{REFERENCES}

Adler, I., Karmarkar, N., Resende, M. and Veiga, G. (1989) An implementation of Karmarkar's algorithm for linear programming. Mathematical programming, 44, 297-335.

Evtushenko, Yu. and Zhadan, V. (1978) A relaxation method for solving problems of nonlinear programming. U.S.S.R. Computational Mathematics and Mathematical Physics, 17, 73-87.

Evtushenko, Yu. and Zhadan, V. (1994) Stable Barrier-projection and Barrier-Newton Methods in Linear Programming. Computational Optimization and Applications, 3, 289-303.

Tanabe K. (1980) A geometric method in nonlinear programming. Journal of Optimization Theory and Applications, 30, 181-210. 